\title{
Lipodystrophy of HIV (LDHIV) in the Head and Neck: Imaging and Clinical Features
}

\author{
Saman Hazany ${ }^{1,2}$, Rafael Rojas ${ }^{1}$, Gul Moonis ${ }^{1}$ \\ ${ }^{1}$ Department of Neuroradiology, Beth Israel Deaconess Medical Center, Boston, USA \\ ${ }^{2}$ Department of Neuroradiology, University of Southern California, Los Angeles, USA \\ Email: saman26@yahoo.com
}

Received April 6, 2012; revised May 19, 2012; accepted June 10, 2012

\begin{abstract}
A subset of HIV-1 infected patients undergoing antiretroviral treatment with HIV-1 protease inhibitors (PI's) develops a syndrome called Lipodystrophy of HIV (LDHIV). LDHIV is characterized by loss of peripheral subcutaneous adipose tissue (face, limbs, buttocks), visceral fat accumulation, and in some cases, lipomatosis in the neck and dorsocervical area .We describe the clinical and imaging features of LDHIV in the head and neck in a series of 5 cases. There is a consistent pattern of fat accumulation in the dorsocervical region with paucity of fat in the face. This classic appearance should be recognized as potentially related to drug toxicity in the HIV infected population.
\end{abstract}

Keywords: Lipodystrophy of HIV; LDHIV; Protease Inhibitor

\section{Introduction}

A subset of HIV-1-infected patients undergoing highly active antiretroviral treatment (HAART), most commonly with HIV-1 protease inhibitors (PIs), develop a lipodystrophy syndrome. Lipodystrophy in Human immunodeficiency virus infected patients (LDHIV) is the most common cause of acquired lipodystrophy in this patient population. It is seen in as many as $40 \%$ of patients treated with protease inhibitors for greater than one year, with more than 100,000 persons affected in the US [1-8]. While LDHIV is a well-recognized entity in the internal medicine literature [3,5-9], its manifestation in the head and neck has not been well described in radiology literature. As diagnosis is usually made on the basis of physical exam and clinical suspicion, imaging is reserved for cases where there is concern for neoplasm. Here we describe imaging features of head and neck involvement in 5 cases of LDHIV and discuss its distinct imaging findings on CT, and MRI which help to differentiate this entity from other causes of head and neck adipose tissue prominence.

\section{Case Series}

A total of 5 patients with LDHIV of the head and neck were identified retrospectively via search of radiology reports and online medical records. Two of the patients had known clinical diagnosis of LDHIV. Four patients presented with a history of neck swelling and or mass. One patient had neck pain. No prior history of lipoma,
Cushing's disease or steroid ingestion was elicited. No adenopathy on examination was appreciated in any patient. All patients had been on combination HAART therapy ranging from 2 months to 15 years from presentation. Additional relevant clinical information is listed in Table 1. Imaging studies included a total of 2 CT's, 4 MRI's and 1 Ultrasound. Histopathologic correlation was available in one patient in whom liposuction was performed for dysphagia (Patient 1).

On MRI and CT all patients had large accumulations of fat with non-enhancing scant septations in a mass like configuration predominantly in the dorsocervical, posterior triangle and submental regions, with sparing of the perivertebral and retropharyngeal spaces (Figures 1 and 2). In one patient there was partial fatty infiltration of bilateral parotid glands. In three patients there was notably scarce fat in the face. There was no evidence of soft tissue mass in any case. Ultrasound was the initial imaging study in patient 1 which revealed a non specific lesion representing either fat or soft tissue deposit and further evaluation with cross sectional imaging was suggested. Liposuction was performed in this patient for dysphagia. Tan-yellow homogeneous adipose tissue without focal hemorrhage or necrosis was seen upon gross examination of the specimen fat. Pathologic evaluation revealed findings most compatible with benign adipose tissue with microscopic fat necrosis and no atypical hyperchromatic stromal cells nor adipocytes with atypical nuclei (Figure 3). 
Table 1. Summary of patients' history and imaging.

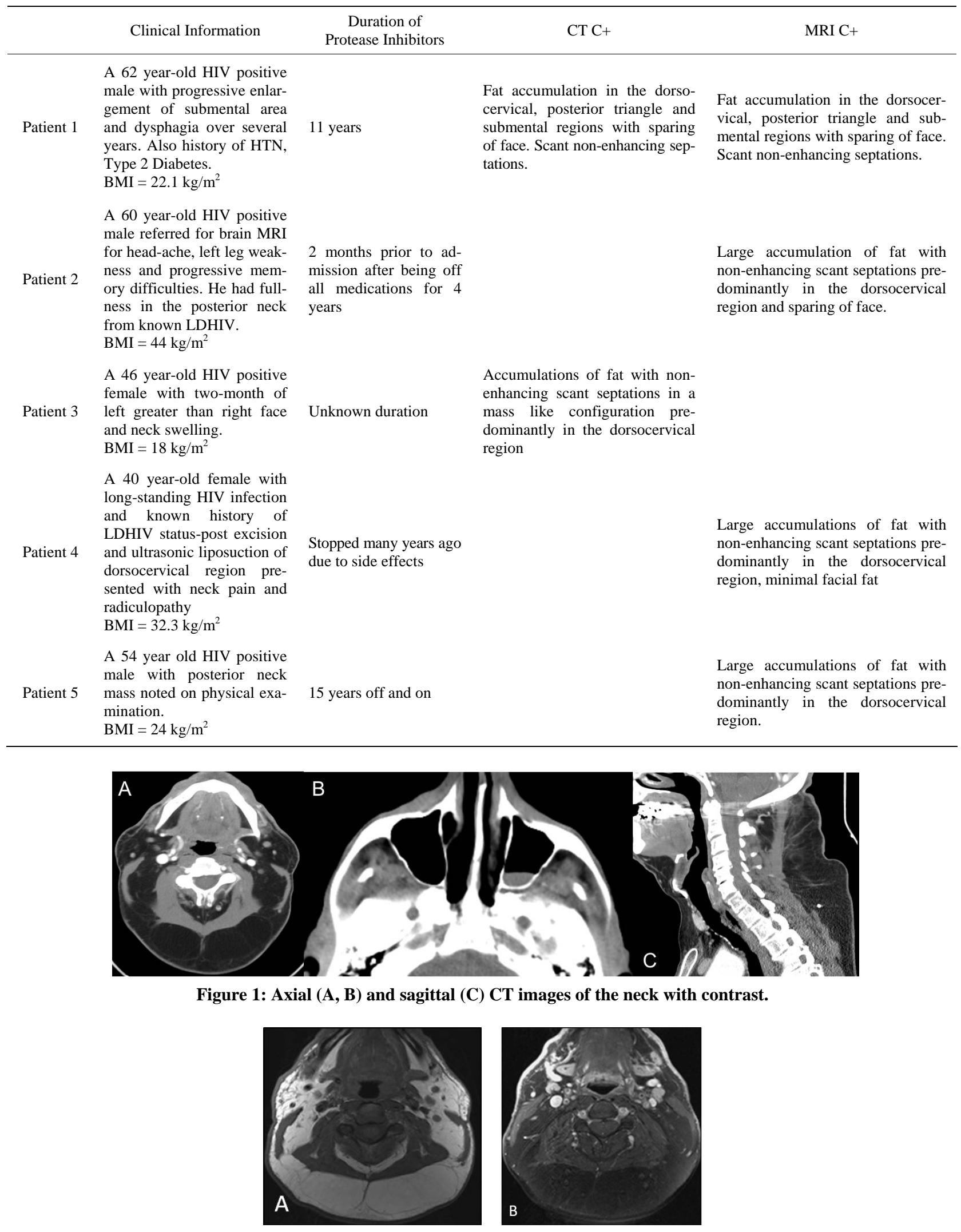

Figure 2. Axial pre-gadolinium (A) and post gadolinium (B) fat saturated T1 weighted images. 


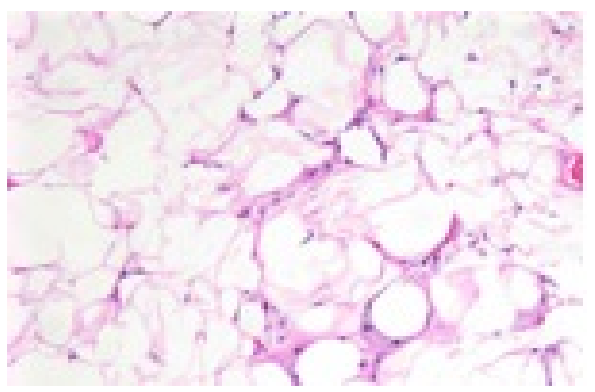

Figure 3. H \& E stain, $\times 200$.

\section{Discussion}

LDHIV is a syndrome seen in the HIV positive population being treated with highly active antiretroviral therapy(HAART). These drugs are classified by the phase of the retrovirus life-cycle that they inhibit. Protease inhibitors (PIs) target viral assembly by inhibiting the activity of protease enzyme used by HIV to cleave nascent proteins for final assembly of new virons. Non protease inhibitors include Nucleoside and nucleotide reverse transcriptase inhibitors (NRTI), Non-nucleoside reverse transcriptase inhibitors (NNRTI), Integrase inhibitors, entry inhibitors (or fusion inhibitors) and maturation inhibitors. Some common PI's used in clinical practise include Indinavir, Ritonavir, Darunavir, Atazanavir, Fosamprenavir, Lopinavir, Nelfinavir, Saquinavir, Tipranavir and Amprenavir.

Carr et al. [10] initially described a syndrome of peripheral lipodystrophy and metabolic abnormalities related to the use of PIs in 1998. LDHIV syndrome is associated with dyslipidemia, impaired glucose tolerance, hyperinsulinemia and insulin resistance [11-13]. Patients with HIV lipodystrophy syndrome are also at increased risk for the development of atherosclerosis and myocardial infarction [14].

The pathogenesis of LDHIV is not entirely clear. A complex interaction of HIV-1 infection, individual subject characteristics such as body weight and baseline lipid level and drug treatment-related events is postulated to trigger the syndrome [3]. Active lipolysis in subcutaneous fat, along with impaired fat storage capacity in the subcutaneous depots drives ectopic deposition of lipids, either in the viscera or in nonadipose sites [3,4,7,15].

While PIs have been the strongest link to LDHIV, other factors, such as duration of HIV infection, age, and gender, may also contribute to the risk of development of LDHIV Besides PI's NRTI's have also been associated with LDHIV [3].

HIV associated Lipodystrophy has two components: lipohypertrophy and lipoatrophy. Lipohypertrophy manifests as enlargement of the dorsocervical fat pad (buffalo hump), circumferential expansion of the neck (double chin), breast enlargement, and abdominal visceral fat accumulation. Lipoatrophy is seen as by peripheral fat wasting with loss of subcutaneous tissue in the face, arms, legs, and buttocks [3,7]. It can be disfiguring and may be confused with neoplasm on physical exam. On imaging LDHIV of the head and neck manifests as accumulation of fat in the dorsocervical region with scant non enhancing septations and relative paucity of subcutaneous fat in the face.

The imaging differential diagnosis of LDHIV in the head and neck includes adipose tissue prominence of obesity, Cushing's syndrome, lipoma, Madelung's neck or lipodystrophies. A characteristic mass like fat deposition in the neck and dorsocervical region and markedly scarce fat in the subcutaneous tissues of the face distinguishes LDHIV from obesity. While pathologic evaluation reveals normal mature adipocytes identical to benign lipoma, its diffuse distribution on imaging excludes lipoma which presents as a mass. Prominence of subcutaneous fat in the dorsocervical and upper thoracic region resembles the "buffalo hump" seen in Cushing syndrome. However, relative facial fat scarcity and clinical history can help differentiate from Cushing's syndrome which has a characteristic "moon face".

Madelung's neck —or benign symmetric lipomatosisis a rare lipodystrophic disease of unknown origin. It presents with painless symmetric unencapsulated fatty deposits diffusely involving the head and neck, shoulder girdle, and upper body. It is most commonly seen in middle-aged men of Mediterranean descent and is associated with a history of alcohol abuse, malignant tumors of the upper airway, neuropathy, diabetes mellitus, hyperlipidemia, and other metabolic disorders [16]. Clinical history and relative scarcity of facial fat should help differentiate Madelung's neck from LDHIV.

Multiple types of hereditary and acquired lipodystrophy have been described including congenital generalized lipodystrophy (Berardinelli-Seip syndrome), familial partial lipodystrophy (Dunnigan type, Kobberling type, mandibuloacral dysplasia type), acquired generalized lipodystrophy (Lawrence syndrome) and acquired partial lipodistrophy (Barraquer-Simons syndrome) [2]. While most of these lipodystrophy syndromes share phenotypic characteristics (including similar adipose tissue distribution and association with metabolic complications, such as insulin resistance) with LDHIV, they generally present in early childhood and adolescence [2]. Al-Attar et al. suggested the use of semi-automated MRI-based adipose tissue quantification in differentiating different types of lipodystrophies [15]. However, clinical history is adequate in distinguishing LDHIV from other types of lipodystrophy in the majority of cases.

While some of our cases did have some subcutaneous facial fat the amount was relatively scarce as compared to fat in the dorsocervical region. In addition, some of 
these patients had BMI's in the range of obesity, which contributed to the deposition of subcutaneous facial fat.

\section{Conclusion}

LDHIV is a common disorder amongst HIV positive population, mostly associated with HAART. Radiologists should be aware of the imaging features of this condition in the head and neck in the HIV positive population because it may mimic a mass. Accurate interpretation can not only exclude neoplasm, but can also alert clinicians to the possible presence of LDHIV-associated metabolic abnormalities.

\section{REFERENCES}

[1] L. R. Gellett, L. Haddon and G. F. Maskell, "CT Appearances of HIV-Related Lipodystrophy Syndrome," British Journal of Radiology, Vol. 74, No. 880, 2001, pp. 382-383.

[2] J. L. Chan and E. A. Oral, "Clinical Classification and Treatment of Congenital and Acquired Lipodystrophy," Endocrine Practice, Vol. 16, No. 2, 2010, pp. 310-323.

[3] D. Chen, A. Misra and A. Garg, "Clinical Review 153: Lipodystrophy in Human Immunodeficiency Virus-Infected Patients,” Journal of Clinical Endocrinology and Metabolism, Vol. 87, No. 11, 2002, pp. 4845-4856. doi:10.1210/jc.2002-020794

[4] S. Walmsley, A. M. Cheung, G. Fantus, et al., "A Prospective Study of Body Fat Redistribution, Lipid, and Glucose Parameters in HIV-Infected Patients Initiating Combination Antiretroviral Therapy," HIV Clinical Trials, Vol. 9, No. 5, 2008, pp. 314-323. doi:10.1310/hct0905-314

[5] V. Pao, G. A. Lee and C. Grunfeld, "HIV Therapy, Metabolic Syndrome, and Cardiovascular Risk," Current Atherosclerosis Reports, Vol. 10, No. 1, 2008, pp. 61-70. doi:10.1007/s11883-008-0010-6

[6] P. C. Tien, S. R. Cole, C. M. Williams, et al., "Incidence of Lipoatrophy and Lipohypertrophy in the Women's Interagency HIV Study," Journal of Acquired Immune Deficiency Syndromes, Vol. 34, No. 5, 2003, pp. 461-466. doi:10.1097/00126334-200312150-00003

[7] P. C. Tien and C. Grunfeld "What Is HIV-Associated Llipodystrophy? Defining Fat Distribution Changes in HIV Infection,” Current Opinion in Infectious Diseases, Vol. 17, No. 1, 2004, pp. 27-32. doi:10.1097/00001432-200402000-00005
[8] M. K. Leow, C. L. Addy and C. S. Mantzoros, "Clinical Review 159: Human Immunodeficiency Virus/Highly Active Antiretroviral Therapy-Associated Metabolic Syndrome: Clinical Presentation, Pathophysiology, and Therapeutic Strategies,” Journal of Clinical Endocrinology and Metabolism, Vol. 88, No. 5, 2003. pp. 1961-1976. doi:10.1210/jc.2002-021704

[9] C. Grunfeld and P. Tien, "Difficulties in Understanding the Metabolic Complications of Acquired Immune Deficiency Syndrome,” Clinical Infectious Diseases, Vol. 37, Suppl. 2, 2003, pp. S43-S46. doi:10.1086/375886

[10] A. Carr, K. Samaras, S. Burton, M. Law, J. Freund, D. J. Chisholm and D. A. Cooper, "A Syndrome of Peripheral Lipodystrophy, Hyperlipidaemia and Insulin Resistance in Patients Receiving HIV Protease Inhibitors," AIDS, Vol. 12, No. 7, pp. F51-F58. doi:10.1097/00002030-199807000-00003

[11] W. Rozenbaum, S. Gharakhanian, Y. Salhi, N. Adda, T. Nguyen, C. Vigouroux and J. Capeau, "Clinical and Laboratory Characteristics of Lipodystrophy in a French Cohort of HIV-Infected Patients Treated with Protease Inhibitors," 1st International Workshop on Adverse Drug Reactions and Lipodystrophy in HIV, San Diego, 26-28 June 1999, p. 20.

[12] A. Carr, K. Samaras, A. Thorisdottir, G. R. Kaufmann, D. J. Chisholm and D. A. Cooper, "Diagnosis, Prediction, and Natural Course of HIV-1 Protease-Inhibitor-Associated Lipodystrophy, Hyperlipidaemia, and Diabetes Mellitus: A Cohort Study,” The Lancet, Vol. 353, No. 9170, 1999, pp. 2093-2099. doi:10.1016/S0140-6736(98)08468-2

[13] E. Martínez, R. Casamitjana, I. Conget and J. M. Gatell, "Protease Inhibitor-Associated Hyperinsulinaemia," AIDS, Vol. 12, No. 15, 1998, pp. 2077-2078. doi:10.1097/00002030-199815000-00023

[14] K. Henry, H. Melroe, J. Huebsch, et al., "Severe Premature Coronary Artery Disease with Protease Inhibitors," The Lancet, Vol. 351, No. 9112, 1998, p. 1328. doi:10.1016/S0140-6736(05)79053-X

[15] S. A. Al-Attar, R. L. Pollex, J. F. Robinson, B. A. Miskie, R. Walcarius, C. H. Little, B. K. Rutt and R. A. Hegele, "Quantitative and Qualitative Differences in Subcutaneous Adipose Tissue Stores across Lipodystrophy Types Shown by Magnetic Resonance Imaging,” BMC Medical Imaging, Vol. 7, No. 3, 2007. doi:10.1186/1471-2342-7-3

[16] R. Salgado, A. Bernaerts, B. Op de Beeck, A. De Schepper and P. Parizel, "Madelung's Neck: Cross-Sectional Imaging Observations," American Journal of Roentgenology, Vol. 182, No. 5, 2004, pp. 1344-1345. 